\title{
SINTOMAS DEPRESSIVOS EM PESSOAS COM LESÃO MEDULAR TRAUMÁTICA CRÔNICA*
}

Raelly Ramos Campos', Maira Di Ciero Miranda², Zuíla Maria de Figueireido Carvalho³, Janaina Vall ${ }^{4}$

\begin{abstract}
RESUMO: Alterações do humor indicativas de estado depressivo são comuns em pessoas com lesão medular, principalmente nos casos em que a incapacidade é permanente. Este estudo transversal foi realizado em 2012, em Fortaleza-Ceará, com 35 pacientes portadores de lesão medular traumática e aplicada a Escala de Rastreamento Populacional para Depressão com o objetivo de avaliar a ocorrência de sintomas depressivos. Entre os participantes 91,43\% eram paraplégicos e em 37,14\% a etiologia da lesão medular foi perfuração por arma de fogo. Quanto aos sintomas depressivos, 48,57\% dos participantes demonstraram ausência de sintomas; 22,86\% apresentaram depressão leve a moderada e 28,57\% apresentaram depressão grave. Conclui-se que a depressão é frequente em portadores de lesão medular traumática crônica; a Escala de Rastreamento é de uso simples e com boa sensibilidade para detecção do transtorno depressivo nestes pacientes.
\end{abstract}

DESCRITORES: Traumatismos da medula espinhal; Depressão; Avaliação.

\section{DEPRESSIVE SYMPTOMS IN PEOPLE WITH CHRONIC SPINAL CORD INJURY}

ABSTRACT: Changes in mood indicative of a depressive state are common in persons with spinal cord injuries, principally in those cases where the disability is permanent. This cross-sectional study was undertaken in 2012, in Fortaleza-Ceará, with 35 patients with traumatic spinal cord injuries, and through applying the Scale for Populational Screening for Depression with the aim of evaluating the occurrence of depressive symptoms. Among the participants, $91.43 \%$ were paraplegics and in $37.14 \%$ the etiology of the spinal cord injury was a gunshot wound. In relation to the depressive symptoms, $48.57 \%$ of the participants showed an absence of symptoms; $22.86 \%$ had mild to moderate depression and $28.57 \%$ had serious depression. It is concluded that depression is frequent in those with chronic traumatic spinal cord injuries; the Screening Scale is simple to use and has good sensitivity for the detection of depressive disorder in these patients.

DESCRIPTORS: Traumatic spinal cord injury; Depression; Evaluation.

\section{SÍNTOMAS DEPRESIVOS EN PERSONAS CON LESIÓN MEDULAR TRAUMÁTICA CRÓNICA}

RESUMEN: Alteraciones del humor indicativas de estado depresivo son comunes en personas con lesión medular, principalmente en los casos en que la incapacidad es permanente. Este estudio transversal fue realizado en 2012, en Fortaleza, Ceará, con 35 pacientes portadores de lesión medular traumática y aplicación de la Escala de Investigación Poblacional para Depresión con el objetivo de evaluar la ocurrencia de síntomas depresivos. Entre los participantes, 91,43\% eran parapléjicos y en el 37,14\% la etiología de la lesión medular fue perforación por arma de fuego. Cuanto a los síntomas depresivos, el 48,57\% de los participantes demostraron ausencia de síntomas; el 22,86\% presentaron depresión leve a moderada y el 28,57\% presentaron depresión grave. Se concluye que la depresión es frecuente en portadores de lesión medular traumática crónica; la Escala de Investigación es de uso simple y con buena sensibilidad para detección del trastorno depresivo en estos pacientes.

DESCRIPTORES: Traumatismos de la médula espinal; Depresión; Evaluación.

*Monografia de conclusão de Curso de Graduação apresentada ao Departamento de Enfermagem da Universidade Federal do Ceará - UFC em 2012.

${ }^{1}$ Enfermeira. Membro do Núcleo de Pesquisa e Extensão em Enfermagem Neurológica - NUPEN UFC.

${ }^{2}$ Enfermeira. Doutora em Enfermagem. Professora do Departamento de Enfermagem UFC. Membro do NUPEN.

${ }^{3}$ Enfermeira. Pós-Doutora em Enfermagem. Professora do Departamento de Enfermagem da UFC. Coordenadora do NUPEN.

${ }^{4}$ Enfermeira. Doutora em Ciências Médicas. Professora do Departamento de Enfermagem da UFC. Membro do NUPEN.

Autor correspondente:

Recebido: 04/12/2012

Raelly Ramos Campos

Aprovado: 17/07/2013

Universidade Federal do Ceará

Rua Alexandre Baraúna, 1115 - 60430-160 - Fortaleza-CE-Brasil

E-mail: raellyramos@hotmail.com 


\section{INTRODUÇÃO}

A lesão medular traumática, completa ou incompleta, ocorre quando um evento traumático, como o associado a acidentes automobilísticos, mergulho, agressão com arma de fogo ou queda, resulta em lesão das estruturas medulares, interrompendo a passagem de estímulos nervosos através da medula. É um grave problema em saúde pública no Brasil, com elevado índice de pessoas com lesão medular, sendo a maioria jovem, com predomínio do sexo masculino e no auge da sua produtividade ${ }^{(1)}$; aproximadamente cinco homens para uma mulher, entre 18 e 40 anos de idade, de etiologia traumática, cuja incidência tem aumentado de maneira significativa nos últimos $20 \operatorname{anos}^{(2)}$.

Os mecanismos de enfrentamento desenvolvidos pelas pessoas portadoras de condições crônicas ao longo de seu processo de evolução é um dos principais focos de interesse na área. Dentre as doenças crônicas da modernidade, a lesão medular traumática é umas das condições de maior impacto no desenvolvimento humano. Esta condição não evolui, necessariamente, para o óbito, mas limita e demanda completa modificação no estilo e nas opções de vida da pessoa acometida ${ }^{(3)}$.

Distúrbios do humor, particularmente depressão, são frequentes em pacientes com lesão medular. A integração precoce em programas de reabilitação e socialização, incluindo atividades esportivas, diminui a prevalência de depressão e ansiedade ${ }^{(4)}$. A depressão, segundo o Manual Diagnóstico e Estatístico de Transtornos Mentais (DSM-IV) ${ }^{(5)}$, é caracterizada, essencialmente, por um período de humor deprimido ou perda de interesse ou prazer por quase todas as atividades.

Assim, surge a problemática: as pessoas com lesão medular estariam sujeitas à depressão de modo mais frequente que a população em geral diante das dificuldades na vida cotidiana devido ao seu estado de dependência? Fez-se necessário, portanto, efetivo dimensionamento do problema entre essas pessoas, com o intuito de melhor subsidiar as equipes de programas de reabilitação.

O uso de escalas de avaliação para identificação e rastreamento de depressão pode ser uma estratégia importante para diminuir o sub diagnóstico deste transtorno, o qual pode ser tratado de modo a amenizar o sofrimento psíquico das pessoas com lesão medular. Neste contexto, o objetivo deste estudo foi avaliar a ocorrência de sintomas depressivos em pessoas com lesão medular traumática crônica.

\section{MÉTODO}

Pesquisa exploratória, descritiva e transversal, com abordagem quantitativa. A pesquisa foi realizada no domicílio de pessoas com lesão medular traumática crônica. A coleta foi realizada no período de janeiro a abril de 2012.

A população representada por pessoas com lesão medular traumática cadastrados em um banco de dados do Núcleo de Pesquisa e Extensão em Enfermagem Neurológica, da Universidade Federal do Ceará, contava com 107 pessoas. Os critérios de inclusão de participantes no estudo foram: serem portadores de lesão medular por mais de seis meses (fase crônica), maiores de 18 anos, de ambos os sexos e residirem no Município de Fortaleza. Os critérios de exclusão foram pessoas com lesão medular não traumática, com déficit cognitivo, seja por dano neurológico ou transtorno psiquiátrico. Assim, obteve-se para amostra deste estudo um total de 35 pessoas.

A coleta dos dados foi realizada mediante a utilização de um formulário com as variáveis de interesse do estudo: dados de identificação do participante; tipo, tempo e causa da lesão medular; alterações psíquicas anteriormente à lesão; enfrentamento da situação atual; e aplicação de um questionário autoavaliativo que foi a Escala de Rastreamento Populacional para Depressão (CES-D).

A CES-D foi desenvolvida com a finalidade de detecção de sintomas depressivos em populações $\operatorname{adultas}^{(6)}$. O desenvolvimento deste instrumento foi baseado em outras escalas de sintomas depressivos e tem sido amplamente utilizada em estudos clínicos e populacionais ${ }^{(6-7)}$. A CES- D é composta de 20 itens de autorelato de depressão que tem o objetivo de detectar sintomas relevantes de depressão na população adulta. Os sintomas são referentes à semana que precede a aplicação da escala ${ }^{(8)}$.

Os itens do inventário referem-se à ocorrência de irritabilidade, diminuição do apetite, desânimo, autodepreciação, falta de concentração, sentir- se deprimido, inibição para o trabalho, pessimismo, sensação de fracasso, sentimento de culpa, medo, distúrbio do sono, sentimento de infelicidade, diminuição da fala, solidão, sentimento de desvalorização, déficit no aproveitamento da vida, crises de choro, tristeza, cansaço e sentimento de retração.

As respostas a cada uma das questões eram dadas segundo a frequência com que cada sintoma esteve presente na semana precedente à aplicação do instru- 
mento, atribuindo uma pontuação que variava de $0 \mathrm{a}$ 3 , onde raramente ou nunca correspondia a pontuação zero; durante pouco ou algum tempo correspondia a pontuação 1; ocasionalmente ou durante um tempo moderado correspondia a pontuação 2 ; e durante a maior parte do tempo ou todo o tempo correspondia a pontuação 3. A pontuação total pode, portanto, variar entre zero e 60 em cada um dos vinte itens.

A CES-D indica três possíveis diagnósticos, definidos a partir da soma total da resposta dos participantes a todos os itens do questionário. A pontuação obtida foi interpretada segundo a gravidade da depressão, utilizando-se os pontos de corte: menor que 15 , sem sintomas de depressão; entre 15 a 21, sintomas leves a moderados de depressão; e maior que 21, alto nível de sintomas depressivos.

A aplicação da escala foi no domicílio da pessoa com lesão medular (paraplégico ou tetraplégico) e realizada de forma individualizada. A escala era autoavaliativa; quando o participante não compreendia a questão, a mesma era relida.

Os dados foram tabulados e analisados de forma descritiva, em frequência absoluta e relativa das variáveis de interesse. Os aspectos éticos preconizados na Resolução 196/96 do Conselho Nacional de Saúde ${ }^{(9)}$, que estabelece as Diretrizes e Normas Regulamentadoras de pesquisas envolvendo seres humanos foram respeitados em todas as etapas do estudo. O presente estudo foi aprovado pelo Comitê de Ética e Pesquisa da UFC, sob protocolo n. 291/11.

\section{RESULTADOS}

As características sociodemográficas dos pacientes $(n=35)$ estão evidenciadas na tabela 1 .

A caracterização dos pacientes com relação ao evento traumático que levou a lesão medular, tempo da lesão e o tipo de déficit motor está especificada na tabela 2.

O resultado da avaliação dos sintomas depressivos nas pessoas com lesão medular crônica, por meio da aplicação da CES-D está disposto na tabela 3.

Em relação à participação social com o nível de depressão, percebeu-se que quem faz algum tipo de atividade física, tem menor tendência a ter depressão, com nove $(52,94 \%)$ casos; seguido das pessoas que trabalhavam, com cinco pessoas $(29,41 \%)$; e três pessoas $(17,65 \%)$ que não trabalhavam e não faziam atividade física. Nos indivíduos com depressão de leve a moderada apenas um $(12,5 \%)$ trabalhava; cinco $(62,5 \%)$
Tabela 1 - Distribuição das variáveis sociodemográficas dos indivíduos entrevistados com lesão medular traumática crônica. Fortaleza, 2012

\begin{tabular}{lcc}
\hline VARIÁVEIS & n & \% \\
\hline Sexo & 27 & 77,14 \\
Masculino & 08 & 22,86 \\
Feminino & & \\
Escolaridade & 02 & 5,71 \\
Analfabeto & 11 & 31,43 \\
Ensino fundamental & 17 & 48,57 \\
Ensino médio & 05 & 14,29 \\
Ensino superior & & \\
Ocupação & 13 & 37,14 \\
Sim & 22 & 62,86 \\
Não & & \\
Estado civil & 19 & 54,29 \\
Solteiro & 12 & 34,29 \\
Casado & 2 & 5,71 \\
Viúvo & 2 & 5,71 \\
Divorciado & & \\
Religião & & \\
Católico & 22 & 62,86 \\
Evangélico & 05 & 14,29 \\
Sem religião & 04 & 11,43 \\
Outros & 04 & 11,43 \\
Renda familiar & & \\
1 salário & 16 & 45,71 \\
2 salário & 09 & 25,71 \\
> 3 salários & 10 & 28,57 \\
Procedência & & \\
Capital & 33 & 94,29 \\
Interior & 02 & 5,71 \\
Idade & Média & Desvio padrão \\
& 39,17 & 14,76 \\
\hline
\end{tabular}

faziam atividade física; e dois (25\%) não trabalhavam e nem faziam atividade física. Nos indivíduos com depressão grave, cinco (50\%) não trabalhavam e não faziam atividade física; quatro (40\%) faziam atividade física; e um (10\%) trabalhava. Ficou constatado que nenhuma situação confere proteção contra a depressão nesses casos de lesão medular crônica, pois em todos os casos havia indivíduos com nível de depressão leve, moderado ou grave.

Quando abordada a questão do apoio familiar, os resultados foram: 30 pacientes $(85,71 \%)$ relataram receber apoio familiar, sendo $14(40 \%)$ solteiros, $12(34,28 \%)$ casados, dois $(5,71 \%)$ viúvos e dois $(5,71 \%)$ divorciados. Somente dentre os solteiros $(n=5)$ houve relato de não se sentirem apoiados na família. 
Tabela 2 - Distribuição das pessoas entrevistadas com lesões medulares de acordo com as causas do evento traumático medular, tipo de déficit motor e tempo de lesão. Fortaleza, 2012

\begin{tabular}{lcc}
\hline \multicolumn{1}{c}{ VARIÁVEIS } & n & \% \\
\hline Déficit motor & & \\
Paraplégico & 32 & 91,43 \\
Tetraplégico & 03 & 8,57 \\
Tempo de lesão & & \\
6 meses a 2 anos & 01 & 2,86 \\
2 a 5 anos & 05 & 14,28 \\
Mais de 5 anos & 29 & 82,86 \\
Causa & & \\
Perfuração com arma de fogo & 13 & 37,14 \\
Acidente & 09 & 25,71 \\
Queda & 07 & 20,00 \\
Mergulho & 02 & 5,71 \\
Outros & 04 & 11,43 \\
\hline
\end{tabular}

Tabela 3 - Distribuição dos indivíduos com lesão medular traumática crônica segundo à pontuação da CES-D. Fortaleza, 2012

\begin{tabular}{lcc}
\hline PONTUAÇÃO NA ESCALA & n & \% \\
\hline$<15$, sem sintomas de depressão & 17 & 48,57 \\
15-21, sintomas leve a moderada & 08 & 22,86 \\
$\begin{array}{l}\text { 21, altos níveis de sintomas } \\
\text { depressivos }\end{array}$ & 10 & 28,57 \\
\hline
\end{tabular}

\section{DISCUSSÃO}

Podemos afirmar que a maior parte das pessoas com lesão medular participantes deste estudo apresentaram sintomas sugestivos de depressão, pois, 18 dos 35 entrevistados obtiveram escores acima de 15 na escala. Quase 1/3 dos entrevistados (28,57\%) estavam vivenciando um episódio depressivo de maior gravidade no período da entrevista, identificando, desta forma, a necessidade de atenção profissional. A avaliação realizada através da aplicação da escala não foi capaz de estimar o tempo de sofrimento psíquico, limitou-se a identificar os casos com sinais de depressão com pontuação acima de 21 e os prováveis, com pontuação entre 15 e 21.

Portanto, a maneira como a enfermidade é percebida, por sua vez, é o resultado, tanto das características intrínsecas da personalidade do enfermo, como também, das características do meio social em que ele vive e, em particular, da maneira como a enfermidade é percebida nesse meio ${ }^{(10)}$.

Baseado em observações não científicas, sabe- -se que a vida com dependência estaria relacionada a elevados índices de depressão, ansiedade, desesperança e tampouco de ideação suicida. No entanto, podemos observar que praticamente a metade dos casos avaliados não apresentou sinais indicativos de depressão. Isso reforça que a existência de depressão estaria mais relacionada às características inerentes a cada indivíduo, tendo caráter individual, não sendo a lesão medular, em si, a determinante na manifestação de quadros depressivos significativos ${ }^{(10)}$. Nem sempre o sofrimento psíquico está inteiramente associado à extensão do dano físico.

Além de humor deprimido ou perda de interesse ou prazer ${ }^{(5)}$, o indivíduo também pode experimentar pelo menos quatro dos sintomas adicionais: alterações no apetite ou peso, sono e atividade psicomotora; diminuição da energia; sentimentos de desvalia ou culpa; dificuldades para pensar, concentrar-se ou tomar decisões, pensamentos recorrentes sobre morte ou ideação suicida, planos ou tentativas de suicídio. O episódio é acompanhado por sofrimento ou prejuízo clinicamente significativo no funcionamento social, profissional ou outras áreas importantes da vida do indivíduo. O número e a gravidade dos sintomas permitem determinar três graus de um episódio depressivo: leve, moderado e grave ${ }^{(11)}$.

No primeiro deles, o paciente usualmente sofre com a presença dos sintomas, mas provavelmente será capaz de desempenhar a maior parte das atividades. No grau moderado, o paciente, aparentemente, tem muita dificuldade para continuar a desempenhar as atividades de rotina. Já na depressão grave, vários dos sintomas são marcantes e angustiantes - tipicamente a perda da auto estima e ideias de desvalia ou culpa; as ideias e os atos suicidas são comuns e observa-se, em geral, uma série de sintomas somáticos. Essa última pode, ainda, ser acompanhada de sintomas psicóticos ${ }^{(11)}$.

Os achados apontam para uma preocupação importante, pois $10(28,57 \%)$ indivíduos avaliados foram classificados como deprimidos graves e $8(22,86 \%)$ com sinais de depressão leve a moderada. Vale ressaltar que todos se encontram na fase crônica da lesão medular, representando que não houve, em nenhum momento, uma abordagem terapêutica visando o controle desse agravo, o que representa uma influência, sobremaneira danosa, sobre a qualidade de vida desses indivíduos por período prolongado.

Em relação ao tempo de lesão ${ }^{(12)}$, durante a fase aguda do trauma, a negação pode ser um mecanismo protetor de defesa dos pacientes, ajudando-os a superar 
a realidade do acontecimento. À medida que ocorre esta fase e eles passam a conscientizarem- se do agravo, o processo de luto pode prolongar-se e tudo se fecha, uma vez que eles passam a reconhecer as metas de longo alcance e as expectativas podem ser interrompidas ou alteradas permanentemente.

A experiência individual mostra que o tempo é diferente para cada um e depende do apoio da família, da formação prévia, da idade, das causas da lesão, entre outros. A capacidade de tocar a vida após a lesão varia muito em cada pessoa ${ }^{(12)}$.

As inúmeras sequelas da lesão medular transformam de forma significativa a vida dos indivíduos, tanto nos aspecto físico, como emocional, ocupacional, social, relacional, valorativo e de auto percepção. Isso pode gerar medo, desesperança e sentimento de inferioridade, o que leva ao isolamento social pela própria dificuldade de expressar emoções e dar continuidade em um processo de busca da independência e autonomia. Associa-se muito a ideia da deambulação como fator determinante para a convivência dos indivíduos na sociedade, como se o aspecto físico fosse determinante para a volta de sua participação no meio social ${ }^{(13)}$.

Em relação à participação social, segundo pesquisadores $^{(12)}$ apenas com a interação social o indivíduo poderá descobrir novas maneiras de locomoção, caminhando na sociedade, reconhecendo que as dificuldades não são empecilho para uma vida social ativa e autônoma. Por outro lado, o ensino ao acompanhante cuidador não deve constituir livre escolha do enfermeiro, mas uma obrigação profissional ligada à qualidade e à responsabilidade pelo cuidado. É, portanto, um compromisso moral ${ }^{(14)}$.

A identificação das incapacidades consequentes da lesão acarretam dificuldades ou impedem o desempenho de uma determinada função e devem ser consideradas na reabilitação ${ }^{(15)}$, uma vez que a dependência e/ ou incapacidade influenciam negativamente o estado psicoemocional do paciente. O enfermeiro precisa conhecer a natureza dos requisitos de autocuidado terapêutico existentes na pessoa com lesão medular, unindo teoria e prática, com vistas a regular o exercício e/ ou o desenvolvimento das capacidades de autocuidado $^{(16)}$, auxiliando na independência e autocuidado com qualidade de vida.

Além da família, para dar continuidade ao cuidado ao paciente após a alta, é preciso haver uma rede de apoio social suficiente e completa para responder às necessidades que demandam da assistência de alta complexidade que exige um paciente com lesão me- dular. Ou seja, uma instituição de retaguarda a fim de garantir a reabilitação precoce, continuidade do cuidado do paciente e inclusão social como processo pelo qual as pessoas com necessidades especiais se preparam para assumir seus papéis na sociedade ${ }^{(17-18)}$.

Considerando que a maioria das pessoas com lesão medular está na juventude ou idade adulta jovem, é vital que a sociedade esteja preparada para ampará-las, permitindo que elas retornem à vida de uma maneira saudável e que, de acordo com sua vontade, sejam estimulados a se sentirem produtivas ${ }^{(12)}$, superando sentimentos que podem ocorrer com frequência, como agressividade, insegurança, ansiedade, impulsividade, isolamento social, desespero, sentimento de inferioridade, ambivalência, raiva, medo e desesperança ${ }^{(13)}$.

Neste momento primeiramente a família, depois os profissionais de saúde e a sociedade devem estar preparados para proporcionar-lhes o apoio que auxilie no enfrentamento desta nova condição e no desejo de seguir a vida em frente, mesmo com as novas limitações e perspectivas ${ }^{(3)}$. Deste modo, a forma como cada indivíduo enfrenta suas limitações é dependente da vida anterior, numa determinação que envolve sua vida anterior a lesão com, família, amigos, história, conhecimento, nível sócio econômico, que vai juntar a vida atual, em que esta pessoa vai se deparar com limitações e possibilidades. Estas necessitam serem vencidas, pois após a lesão medular, a vida será completamente diferente da anterior, desconhecida, com novos questionamentos e prioridades e, para vivê-la, se utiliza de todo arcabouço afetivo de sua história.

\section{CONCLUSÃO}

As escalas de avaliação da depressão ajudam na detecção dos sintomas depressivos e na elaboração do próprio diagnóstico, além de auxiliarem o acompanhamento do paciente e o resultado dos tratamentos.

A aplicação da CES-D para pacientes com lesão medular traumática crônica no domicílio utilizada neste estudo foi eficaz para identificar a ocorrência de depressão nos lesionados medulares crônicos, que muitas vezes passa despercebida pela família e pelo profissional de saúde que deixam de acompanhar esses pacientes depois do tratamento agudo da lesão.

Considerando que número significativo de pessoas com lesão medular é de jovens e esses terão que atravessar muitos anos com a deficiência, não se pode permitir que a depressão seja um fator contributivo para a qualidade de vida precária dos paraplégicos e 
tetraplégicos. O acompanhamento sistemático dessa clientela deveria ser efetuado dentro do serviço de saúde para garantir efetiva assistência global das necessidades proporcionando, além da reabilitação física, a psicológica e social.

A avaliação de sintomas depressivos nesses indivíduos se mostrou de grande importância, pois, verificou-se que é de ocorrência frequente nesse tipo de clientela, e que exige maior sensibilização por parte dos familiares e profissionais de saúde na detecção do problema e na busca de uma solução terapêutica eficaz.

\section{REFERÊNCIAS}

1. Gonçalves AMT, Rosa LN, D' Angelo CT, Savordelli CL, Bonin GL, Squarcino IM, et al. Aspectos epidemiológicos da lesão medular traumática na área de referencia do Hospital Estadual Mario Covas. Arq. méd. ABC. 2007;32(2):64-6.

2. Paz LM, Rodríguez MR, Cossio JG, Quinteiro IE. Accionar de enfermería y aplicación del Índice de Barthel a pacientes lesionados medulares en la clínica raquimedular del CIREN. Medwave. 2005;5(10). (Não tem livre acesso da versão em pdf para verificar as páginas do artigo)

3. Murta SG, Guimarães SS. Enfrentamento à lesão medular traumática. Estudos de Psicologia 2007;12(1):57-63.

4. Rede Sarah De Hospitais De Reabilitação. Mapa da morbidade por causas externas- 2004. [Internet] 2004 [acesso em 27 ago 2011]. Disponível: www.sarah.br.

5. American Psychiatric Association: Diagnostic and Statistical Manual of Mental Disorders, $4^{\mathrm{a}}$ ed. Washington (DC): American Psychiatric Association; 1994.

6. Radloff LS. The CES-D Scale: a self-report depression scale for research in general population. Applied Psychological Measurement. 1977;1(3):385-401.

7. Roberts RE, Vernon SW. The center for Epidemiologic Studies Depression Scale: its use in a community sample. Am J Psychiatry. 1983;140(1):41-6.

8. Silveira D, Jorge M. Propriedades Psicométricas da Escala de rastreamento para Depressão CES-D em populações clínica e não-clínica de adolescentes e adultos jovens. Rev. psiquiatr. clín. 1998;25(5):251-61.

9. Ministério da Saúde (BR). Conselho Nacional de Saúde. Resolução n. 196 de 10 de outubro de 1996. Diretrizes e normas regulamentadoras de pes $\neg$ quisa em seres humanos. Brasília; 1996.

10. Berto CD, Barreto DBM. Pessoas com lesão medular traumática: as alterações biopsicossociais e as expectativas vividas. Unoesc \& Ciência - ACHS, Joaçaba. 2011;2(2):174-83.

11. Dornelles C. DSM-IV-TR - Manual Diagnóstico e Estatístico de Transtornos Mentais. $4^{\mathrm{a}}$ ed. Porto Alegre: Artmed; 2002.

12. Schoeller SD, Bitencourt RN, Leopardi MT, Pires DP, Zanini MTB. Mudanças na vida das pessoas com lesão medular adquirida. Rev. Eletr. Enf. 2012;14(1):95-103.

13. Fechio MB, Pacheco KMB, Kaihami HN, Alves VLR. A repercussão da lesão medular na identidade do sujeito. Acta Fisiátr. 2009;16(1):38-42.

14. Carvalho ZMF, Holanda KM, Freitas GL, Silva GA. Pacientes com lesão raquimedular: experiência de ensino-aprendizagem do cuidado para suas famílias. Esc. Anna Nery. 2006; 10(2):316-22.

15. Vall J, Lemos KIL, Janebro ASI. O processo de reabilitação de pessoas portadoras de lesão medular baseado nas teorias de enfermagem de Wanda Horta, Dorothea Orem e Callista Roy: um estudo teórico. Cogitare enferm. 2005;10(3):63-70.

16. Brito MAGM, Bachion MM, Souza JT. Diagnósticos de enfermagem de maior ocorrência em pessoas com lesão medular no contexto do atendimento ambulatorial mediante abordagem baseada no modelo de Orem. Rev. Eletr. Enf. 2008;(10):13-28.

17. Nogueira PC. Sobrecarga do cuidado e qualidade de vida relacionada à saúde de cuidadores de indivíduos com lesão medular [tese]. Ribeirão Preto (SP): Universidade de São Paulo; 2010.

18. Feijó ARA. Pessoa portadora de deficiência: direitos humanos e proteção jurídica da pessoa portadora de deficiência. Brasília: Ministério da Justiça, Secretaria de Estado dos Direitos Humanos; 2003. 